\title{
Pegfilgrastim Maintains Relative Dose Intensity and Decreases Hospitalisations in Patients With Endometrial Cancer
}

\author{
KENRO CHIKAZAWA, KEN IMAI, TAKAKI ITO, AZUSA KIMURA, KO HIROYOSHI, \\ YOKOTA MIHO, TOMOYUKI KUWATA and RYO KONNO
}

Department of Obstetrics and Gynecology, Jichi Medical University, Saitama Medical Center, Saitama, Japan

\begin{abstract}
Background/Aim: Data are limited regarding the use of pegfilgrastim in gynaecologic oncology. We evaluated its efficacy for maintaining dose intensity during chemotherapy. Patients and Methods: We retrospectively examined the data of 65 women (26 pegfilgrastim users) who underwent primary surgical treatment for stages IB-IV endometrial cancer and had adjuvant chemotherapy containing platinum and taxane; the primary outcome was a relative dose intensity $\geq 85 \%$. Results: In the pegfilgrastim $v s$. the control group, body mass index (26.6 $\pm 5.9 \mathrm{vs}$. $23.4 \pm 4.4$ ), rate of relative dose intensity $\geq 85 \%$ ( $88.5 \% \mathrm{vs}$. $15.4 \%)$, plus other adverse event incidences were significantly higher; rate of neutropenia, total hospital visits during chemotherapy $(11.0 \pm 2.1 \quad$ vs. $18 \pm 5.6$ days $)$, unscheduled hospital visits (1.1 1.8 vs. $5.8 \pm 5.1$ days), and unscheduled granulocyte colony-stimulating factor injections $(0.58 \pm 1.7$ vs. $6.4 \pm 5.1$ days) were significantly lower. Conclusion: Pegfilgrastim can maintain a dose intensity of $\geq 85 \%$ during chemotherapy for the treatment of gynaecologic cancers and decrease hospital-visit frequency.
\end{abstract}

The relative dose intensity is the total dose of a chemotherapeutic drug that is compared against a standard dose and schedule across the total duration of chemotherapy. Relative dose intensity has been suggested as a prognostic factor in several cancers $(1,2)$. Relative dose intensity is considered an important predictive factor even in the survival of patients with metastatic solid tumours (3). In gynaecologic oncology, there is a published report on ovarian cancer and

This article is freely accessible online.

Correspondence to: Kenro Chikazawa (ORCiD: 0000-0002-88394483), Department of Obstetrics and Gynaecology, Saitama Medical Center, The Jichi Medical University, 1-847, Amanuma-Cho, Omiya-Ku, Saitama 330-8503, Japan. Tel: +81 486472111, Fax: +81486485188, e-mail: kendokenro@hotmail.com

Key Words: Pegfilgrastim, endometrial cancer, neutropenia, hospitalisation. relative dose intensity, wherein reduced relative dose intensity for ovarian cancer was a risk factor of overall survival (OS) (1). The abovementioned study was a retrospective study among patients with ovarian cancer; however, there is strong evidence of an association between relative dose intensity and OS in breast cancer or aggressive lymphoma, and granulocyte colony-stimulating factor (G-CSF) improved the chances of attaining a higher relative dose intensity (2). In another recent study, G-CSF was reported to support a relative dose intensity of $85 \%$ or higher (4).

Pegfilgrastim is a long-acting G-CSF that has the most widespread application for approved indications, and supports a relative dose intensity greater than $85 \%$ in patients with lung and breast cancer $(5,6)$. However, there are limited data on the use of pegfilgrastim in gynaecologic oncology. There are limited regimens specified in the National Comprehensive Cancer Network guidelines on chemotherapy for uterine neoplasms (7). Therefore, the relative dose intensity of the first chemotherapy could be crucial to patient outcome and survival.

This study was conducted to evaluate the efficacy of pegfilgrastim in the maintenance of relative dose intensity as adjuvant therapy for endometrial cancer. Moreover, we investigated the utility of pegfilgrastim in reducing hospital visits in patients with endometrial cancer.

\section{Patients and Methods}

This single-centre retrospective study was conducted among women who underwent primary surgical treatment for uterine neoplasms of stages IB-IV [International Federation of Gynecology and Obstetrics (FIGO)] at the Saitama Medical Center, Jichi Medical University, Saitama, Japan between 2014 and 2018. The study inclusion criteria were age over 20 years and treatment with adjuvant chemotherapy containing platinum and taxane. The exclusion criteria were a history of other cancers or the use of a single-agent adjuvant chemotherapy. In this study, patients were assigned into two groups: the pegfilgrastim group and the historical control group. Of the 65 patients eligible for study inclusion, 26 patients routinely received pegfilgrastim, and the remaining patients did not receive pegfilgrastim during their treatment. Pegfilgrastim use was initiated from January 2015 at the study centre. Patients were treated with 
Table I. Patient characteristics of the study population.

\begin{tabular}{lccc}
\hline & $\begin{array}{c}\text { Pegfilgrastim } \\
\text { group } \\
\mathrm{N}=26\end{array}$ & $\begin{array}{c}\text { Non-pegfilgrastim } \\
\text { group } \\
\mathrm{N}=39\end{array}$ & $p$-Value \\
\hline Age & $61.5 \pm 9.7$ & $62.3 \pm 11.3$ & 0.59 \\
Body mass index & $26.6 \pm 5.9$ & $23.4 \pm 4.4$ & $0.016^{*}$ \\
Stage, N (\%) & & & 0.095 \\
I & $11(42.3)$ & $27(69.2)$ & \\
II & $4(15.4)$ & $5(12.8)$ & \\
III & $7(26.9)$ & $6(15.4)$ & \\
IV & $4(15.4)$ & $1(2.6)$ & 0.27 \\
Histopathology, N (\%) & & & \\
Endometrioid & $19(73.1 \%)$ & $31(79.5)$ & \\
Serous & $4(15.4 \%)$ & $1(2.6 \%)$ & \\
Clear cell & $1(3.8 \%)$ & $3(7.7 \%)$ & \\
Carcinosarcoma & $2(7.7 \%)$ & $4(10.3 \%)$ & \\
\hline
\end{tabular}

${ }^{*} p$-value $<0.05$ indicates significance.

chemotherapy in accordance with the guidelines of the Japan Society of Gynecologic Oncology (8). The study protocol was approved by the institutional review board of the Jichi Medical University (S19-127). The requirement for informed consent was waived by the institutional ethics body because of the retrospective nature of the study and a comprehensive written consent had been pre-obtained, for the use of patient data for research purposes.

Two chemotherapeutic regimens were used in the study population: [1] paclitaxel $175 \mathrm{mg} / \mathrm{m}^{2}$ plus carboplatin that was dosed to an area under the curve of concentration-time curve of 6 every 3 weeks; or [2] docetaxel $75 \mathrm{mg} / \mathrm{m}^{2}$ plus carboplatin dosed to an area under the curve of concentration-time curve of 6 every 3 weeks, in patients anxious about developing peripheral neuropathy or adverse events with the paclitaxel-carboplatin regimen. Patients routinely received intravenous injections of pegfilgrastim from January 2015, when it became available for use at the hospital. Patients routinely underwent follow-up laboratory investigations 14 days after the administration of chemotherapy, regardless of whether pegfilgrastim was used. As part of the routine institutional policy, patients received a G-CSF injection if Grade 3 or higher neutropenia was detected.

We recorded the demographics and clinical characteristics of the study participants including body mass index, treatment regimens, FIGO staging, and histological type of the tumour. The primary outcome was a relative dose intensity of $85 \%$ or greater. The secondary outcomes were adverse events such as neutropenia, anaemia, thrombocytopenia, increased serum levels of creatinine and aspartate aminotransferase, peripheral sensory neuropathy, nausea/vomiting, and infections, which were graded in accordance with the criteria specified in the Common Terminology Criteria for Adverse Events version 5.0.

All statistical analyses were undertaken in JMP, version 10.0.0 (for Windows; SAS Institute Japan, Minato, Japan). Demographic variables were reported as mean and standard deviation, whereas data with non-normal distribution were reported as median and range. We used the Kolmogorov-Smirnov variables test to assess normality of distribution of the continuous variables. For variables with normal distribution, continuous data were compared with the ANOVA test. For variables with non-normal distribution, data were
Table II. Pegfilgrastim and relative dose intensity in the study groups.

\begin{tabular}{lccc}
\hline RDI & $\begin{array}{c}\text { Pegfilgrastim } \\
\text { group } \\
\mathrm{N}=26\end{array}$ & $\begin{array}{c}\text { Non-pegfilgrastim } \\
\text { group } \\
\mathrm{N}=39\end{array}$ & $p$-Value \\
\hline$\geq 85 \%$ & $23(88.5 \%)$ & $6(15.4 \%)$ & $<0.001^{*}$ \\
$<85 \%$ & $3(11.5 \%)$ & $33(84.6 \%)$ & \\
\hline
\end{tabular}

RDI: Relative dose intensity. ${ }^{*} p$-value $<0.05$ indicates significance.

compared using the Wilcoxon signed-rank test. Fisher's exact test was used to study the associations between all demographic parameters. For all statistical tests, a two-sided $p$-value less than 0.05 was considered indicative of statistical significance. For the hazard ratio, we estimated $95 \%$ confidence intervals (95\% CIs). Based on our previous research (unpublished findings), we estimated a sample size of 15 patients in both groups and an overall study population of 30 patients, wherein $80 \%$ of patients in the pegfilgrastim group would be expected to have a relative dose intensity of $85 \%$ or higher, and $30 \%$ of patients in the non-pegfilgrastim group would have a relative dose intensity of $30 \%$ or higher.

\section{Results}

A total of 65 patients (age, $62.4 \pm 10.6$ years) were included in the study. Patients were divided into a pegfilgrastim (routinely received pegfilgrastim; $\mathrm{n}=26$; age $61.5 \pm 9.7$ years) and a non-pegfilgrastim arm (did not receive pegfilgrastim; $\mathrm{n}=39 ; 62.3 \pm 11.3$ years). There was no significant betweengroup difference in age $(p=0.59)$, but patients in the pegfilgrastim group had significantly higher body mass index versus the non-pegfilgrastim group $(26.6 \pm 5.9$ vs. $23.4 \pm 4.4$, respectively; $p=0.016)$. The other patient demographics and clinical characteristics are presented in Table I. In the pegfilgrastim group, one of the patients used docetaxelcarboplatin, because of anxiety regarding peripheral neuropathy, and another patient switched to the docetaxelcarboplatin treatment because of symptomatic peripheral neuropathy. In the non-pegfilgrastim group, two patients used docetaxel-carboplatin because of anxiety about peripheral neuropathy, and one patient switched to docetaxelcarboplatin chemotherapy after developing symptoms of peripheral neuropathy. All other patients were treated with a paclitaxel-carboplatin regimen.

In the pegfilgrastim group, the rate of relative dose intensity of $85 \%$ or greater was significantly higher $(88.5 \%$ vs. $15.4 \%$; Table II) than estimated. Three patients in this group achieved relative dose intensity of less than $85 \%$ because of Grade 3 thrombocytopenia.

Other causes for a relative dose intensity of less than $85 \%$ in these patients were neutropenia or infection.

The pegfilgrastim group had a significantly lower reported adverse event rate for neutropenia, although the rate of other 
Table III. Adverse events in the study population.

\begin{tabular}{lccc}
\hline Adverse events & $\begin{array}{c}\text { Pegfilgrastim } \\
\text { group } \\
\mathrm{N}=26\end{array}$ & $\begin{array}{c}\text { Non-pegfilgrastim } \\
\text { group } \\
\mathrm{N}=39\end{array}$ & $p$-Value \\
\hline $\begin{array}{l}\text { Neutropenia, } \\
\text { Grade } \geq 3\end{array}$ & $3(11.5 \%)$ & $36(92.3 \%)$ & $<0.001^{*}$ \\
$\begin{array}{l}\text { Anaemia, Grade } \geq 2 \\
\text { Thrombocytopenia, } \\
\text { Grade } \geq 2\end{array}$ & $5(19.2 \%)$ & $3(7.7 \%)$ & 0.17 \\
$\begin{array}{l}\text { Elevated serum } \\
\text { aspartate } \\
\text { aminotransferase, } \\
\text { Grade } \geq 1\end{array}$ & $6(23.1 \%)$ & $17(43.6 \%)$ & 0.057 \\
$\begin{array}{c}\text { Elevated serum } \\
\text { creatinine, Grade } \geq 1\end{array}$ & 0 & $1(2.6 \%)$ & 0.09 \\
$\begin{array}{l}\text { Nausea/vomiting, } \\
\text { Grade } \geq 1\end{array}$ & $3(11.5 \%)$ & $1(2.6 \%)$ & 0.14 \\
Infections & 0 & $10(25.6)$ & $0.005^{*}$ \\
\end{tabular}

${ }^{*} p$-value $<0.05$ indicates significance.

adverse events was higher in the pegfilgrastim group (Table III). In the non-pegfilgrastim group, 10 patients had infections, and recovered with antibiotic therapy.

Furthermore, total hospital visits for chemotherapy, unscheduled hospital visits due to infection or for receiving additional doses of G-CSF if required, and unscheduled GCSF injections were significantly lower in the pegfilgrastim group $(11.0 \pm 2.1$ vs. $18 \pm 5.6$ days, $p \leq 0.001 ; 1.1 \pm 1.8 v s$. $5.8 \pm 5.1$ days, $p<0.001$; and $0.58 \pm 1.7$ vs. $6.4 \pm 5.1$ days, $p<0.001$, respectively; Table IV).

\section{Discussion}

This study presents the first report of the efficacy of pegfilgrastim in maintaining the relative dose intensity in the chemotherapeutic management of endometrial cancer. Pegfilgrastim not only maintained the relative dose intensity but also decreased the number of hospital visits.

In patients with endometrial cancer, pegfilgrastim effectively maintained the relative dose intensity during adjuvant chemotherapy. In gynaecologic oncology, the reduced relative dose intensity in patients with ovarian cancer is a risk factor for low OS (1). In other cancers too, reduced relative dose intensity is a risk factor for low OS (2). Pegfilgrastim is a long-acting G-CSF that is approved for most of the indications of G-CSF therapy, and supports the maintenance of relative dose intensity above $85 \%$ in patients with lung and breast cancer $(5,6)$. Our study found similar efficacy of pegfilgrastim in the maintenance of a relative dose intensity of $85 \%$ or greater and recommends the use of adjuvant therapy with pegfilgrastim in the field of gynaecologic oncology.
Table IV. Frequency of hospital visits in the study population.

\begin{tabular}{lccc}
\hline Hospital visits & $\begin{array}{c}\text { Pegfilgrastim } \\
\text { group } \\
\mathrm{N}=26\end{array}$ & $\begin{array}{c}\text { Non-pegfilgrastim } \\
\text { group } \\
\mathrm{N}=39\end{array}$ & $p$-Value \\
\hline $\begin{array}{l}\text { Total visits during } \\
\text { chemotherapy }\end{array}$ & $11.0 \pm 2.1$ & $18 \pm 5.6$ & $<0.001^{*}$ \\
$\begin{array}{l}\text { Unscheduled visits } \\
\text { for additional } \\
\text { injection of G-CSF, } \\
\text { or becauseof infection }\end{array}$ & $1.1 \pm 1.8$ & $7.8 \pm 5.1$ & $<0.001^{*}$ \\
$\begin{array}{l}\text { Unscheduled } \\
\text { G-CSF injection }\end{array}$ & $0.58 \pm 1.7$ & $6.4 \pm 5.1$ & $<0.001^{*}$ \\
\hline
\end{tabular}

${ }^{*} p$-value $<0.05$ indicates significance.

Pegfilgrastim was also found to be useful in decreasing hospital visits. In Japan, approximately $30 \%$ of the cancer patients belong to the working age group (9). It has been reported that $64.5 \%$ of the patients find it difficult to continue biweekly hospital visits (10). In addition, approximately $77.4 \%$ of patients experienced difficulty in taking leave from work. A review article has reported that the use of pegfilgrastim is recommended along with chemotherapy, in cancer patients (11). Furthermore, in a meta-analysis, the risk of severe neutropenia was found to be reduced with pegfilgrastim (12).

The rate of neutropenia was significantly lower in the pegfilgrastim group; however, the incidence of other adverse events tended to be higher in this group. The first and subsequent cycles of pegfilgrastim in patients with breast cancer markedly reduced the incidence of febrile neutropenia and febrile neutropenia-related hospitalisations (13). Therefore, although the incidence of neutropenia reduced and the relative dose intensity was maintained, the incidence of other adverse events appeared to increase. This is similar to the effects seen with dose-dense chemotherapy, where patients often need blood transfusions and delay chemotherapy cycles because of anaemia and thrombocytopenia (14). However, in our study, only three patients in the pegfilgrastim group developed thrombocytopenia. Other patients in the pegfilgrastim group achieved a relative dose intensity of $85 \%$ or greater. Our regimens were conventional and not dosedense regimens, such as those reported in the study by Katsumata et al. (14). Therefore, the usual regimens could maintain a relative dose intensity with therapeutic feasibility and a decreased incidence of neutropenia and infection, using adjuvant pegfilgrastim therapy.

At present, the cost factor poses a major economic burden in cancer treatment (15-21). Cancer patients are unable to work due to poor health and end up with reduced work productivity (15-18). The financial implications of cancer 
may further increase as constraints on working capacity reduce employment-based health insurance options and limit the patients' resources for healthcare. These factors together may contribute to the phenomenon of financial ramifications of cancer treatment. Therefore, our study proposes a possible solution to manage these problems.

Our study suggests that pegfilgrastim assists in decreasing the number of hospital visits. This could be helpful in minimising face-to-face contact between the medical staff and patients, which is an ongoing need in the current COVID-19 pandemic situation (22).

Our study's limitations are as follows: 1) all limitations and risk of bias inherent to retrospective studies; 2) a small sample size; 3) potential selection bias, considering that all subjects were recruited from a single institution; and 4) the results may neither be extrapolated nor generalised to other populations.

In conclusion, pegfilgrastim was useful in the maintenance of a relative dose intensity in the required therapeutic range in the treatment of endometrial cancer. Moreover, pegfilgrastim decreased the number of hospital visits.

\section{Conflicts of Interest}

K. Chikazawa received lecture honoraria from Ethicon (Tokyo, Japan), Terumo (Tokyo, Japan), and Chugai Pharmaceutical Co. (Tokyo, Japan). R. Konno received research funds from Yakult Pharmaceutical Industry Co. (Tokyo, Japan) and Chugai Pharmaceutical Co. (Tokyo, Japan), and lecture honoraria from Japan Vaccine Co. (Tokyo, Japan), MSD Japan (Tokyo, Japan), and Chugai Pharmaceutical Co. (Tokyo, Japan). The funding organisations did not contribute to any aspect of conducting or reporting of this study. The other co-authors have no conflict of interests to declare regarding this study.

\section{Authors' Contributions}

K. Chikazawa conceptualized the study and planned the study design. K. Chikazawa and T. Ito undertook data collection and analysis. K. Chikazawa conducted statistical analysis. K. Chikazawa drafted the manuscript. T. Kuwata and R. Konno reviewed and finalized the manuscript. K. Chikazawa and K. Imai drafted the initial and final manuscript. A. Kimura and K. Hiroyoshi collected data. Y. Miho analyzed data. T. Kuwata and R. Konno reviewed and edited the final manuscript.

\section{References}

1 Hanna RK, Poniewierski MS, Laskey RA, Lopez MA, Shafer A, Van Le L, Crawford J, Dale DC, Gehrig PA, Secord AA, Havrilesky LJ and Lyman GH: Predictors of reduced relative dose intensity and its relationship to mortality in women receiving multi-agent chemotherapy for epithelial ovarian cancer. Gynecol Oncol 129(1): 74-80, 2013. PMID: 23262376. DOI: 10.1016/j.ygyno.2012.12.017

2 Wildiers $\mathrm{H}$ and Reiser $\mathrm{M}$ : Relative dose intensity of chemotherapy and its impact on outcomes in patients with early breast cancer or aggressive lymphoma. Crit Rev Oncol Hematol 77(3): 221-240, 2011. PMID: 20227889. DOI: 10.1016/j. critrevonc.2010.02.002

3 Havrilesky LJ, Reiner M, Morrow PK, Watson H and Crawford $\mathrm{J}$ : A review of relative dose intensity and survival in patients with metastatic solid tumors. Crit Rev Oncol Hematol 93(3): 203-210, 2015. PMID: 25459671. DOI: 10.1016/j.critrevonc. 2014.10.006

4 Pérez-Fidalgo JA, Bermejo B, Chirivella I, Martínez MT, González I, Cejalvo JM, Catoira I, Martínez P, Contel E and Lluch A: Retrospective analysis of the use of G-CSF and its impact on dose response for anthracycline plus taxane-based schedules in early breast cancer. Clin Transl Oncol 16(9): 814822, 2014. PMID: 24532305. DOI: 10.1007/s12094-013-1153-7

5 Kanbayashi Y, Ishikawa T, Kanazawa M, Nakajima Y, Kawano R, Tabuchi Y, Yoshioka T, Ihara N, Hosokawa T, Takayama K, Shikata K and Taguchi T: Predictive factors in patients eligible for pegfilgrastim prophylaxis focusing on RDI using ordered logistic regression analysis. Med Oncol 35(4): 55, 2018. PMID: 29549536. DOI: $10.1007 / \mathrm{s} 12032-018-1116-5$

6 Nagashima Y, Chijimatsu H, Furuya K, Kondo J, Maeda Y, Somura H, Takemoto N, Yahara N, Abe T, Hayashi H, Kubo H, Yamamoto $\mathrm{S}$ and Nagano H: Effect of pegfilgrastim primary prophylactic administration on relative dose intensity (RDI) in postoperative adjuvant chemotherapy (TC therapy) for breast cancer - a single-center, retrospective study. Gan To Kagaku Ryoho 44(13): 2087-2090, 2017. PMID: 29361623.

7 Nagase S, Katabuchi H, Hiura M, Sakuragi N, Aoki Y, Kigawa J, Saito T, Hachisuga T, Ito K, Uno T, Katsumata N, Komiyama S, Susumu N, Emoto M, Kobayashi H, Metoki H, Konishi I, Ochiai K, Mikami M, Sugiyama T, Mukai M, Sagae S, Hoshiai H, Aoki D, Ohmichi M, Yoshikawa H, Iwasaka T, Udagawa Y, Yaegashi $\mathrm{N}$ and Japan Society of Gynecologic Oncology: Evidence-based guidelines for treatment of uterine body neoplasm in Japan: Japan Society of Gynecologic Oncology (JSGO) 2009 Edition. Int J Clin Oncol 15(6): 531-542, 2010. PMID: 21069552. DOI: 10.1007/s10147-010-0138-6

8 National Cancer Center, Cancer Information Service. Available at: https://ganjoho.jp/public/index.html [Last accessed July 11, 2020]

9 Cabinet Office's Public Opinion Poll on Cancer. Available at: https://survey.gov-online.go.jp/h28/h28-gantaisaku/index.html [Last accessed July 11, 2020]

10 Aapro M, Boccia R, Leonard R, Camps C, Campone M, Choquet S, Danova M, Glaspy J, Hus I, Link H, Sliwa T, Tesch $\mathrm{H}$ and Valero V: Refining the role of pegfilgrastim (a long-acting G-CSF) for prevention of chemotherapy-induced febrile neutropenia: consensus guidance recommendations. Support Care Cancer 25(11): 3295-3304, 2017. PMID: 28842778. DOI: 10.1007/s00520-017-3842-1

11 Bond TC, Szabo E, Gabriel S, Klastersky J, Tomey O, Mueller U, Schwartzberg L and Tang B: Meta-analysis and indirect treatment comparison of lipegfilgrastim with pegfilgrastim and filgrastim for the reduction of chemotherapy-induced neutropenia-related events. J Oncol Pharm Pract 24(6): 412-423, 2018. PMID: 28614980. DOI: 10.1177/1078155217714859

12 Vogel CL, Wojtukiewicz MZ, Carroll RR, Tjulandin SA, Barajas-Figueroa LJ, Wiens BL, Neumann TA and Schwartzberg LS: First and subsequent cycle use of pegfilgrastim prevents febrile neutropenia in patients with breast cancer: a multicenter, 
double-blind, placebo-controlled phase III study. J Clin Oncol 23(6): 1178-1184, 2005. PMID: 15718314. DOI: 10.1200/ JCO.2005.09.102

13 Katsumata N, Yasuda M, Takahashi F, Isonishi S, Jobo T, Aoki D, Tsuda H, Sugiyama T, Kodama S, Kimura E, Ochiai K, Noda $\mathrm{K}$ and Japanese Gynecologic Oncology Group: Dose-dense paclitaxel once a week in combination with carboplatin every 3 weeks for advanced ovarian cancer: a phase 3, open-label, randomised controlled trial. Lancet 374(9698): 1331-1338, 2009. PMID: 19767092. DOI: 10.1016/S0140-6736(09)61157-0

14 Ekwueme DU, Yabroff KR, Guy Jr GP, Banegas MP, de Moor JS, Li C, Han X, Zheng Z, Soni A, Davidoff A, Rechis R, Virgo $\mathrm{KS}$ and Centers for Disease Control and Prevention (CDC): Medical costs and productivity losses of cancer survivors United States, 2008-2011. MMWR Morb Mortal Wkly Rep 63(23): 505-510, 2014. PMID: 24918485.

15 Guy Jr GP, Ekwueme DU, Yabroff KR, Dowling EC, Li C, Rodriguez JL, de Moor JS, and Virgo KS: Economic burden of cancer survivorship among adults in the United States. J Clin Oncol 31(30): 3749-3757, 2013. PMID: 24043731. DOI: 10.1200/JCO.2013.49.1241

16 Guy GP, Yabroff KR, Ekwueme DU, Smith AW, Dowling EC, Rechis R, Nutt S and Richardson LC: Estimating the health and economic burden of cancer among those diagnosed as adolescents and young adults. Health Aff (Millwood) 33(6): 1024-1031, 2014. PMID: 24889952. DOI: 10.1377/hlthaff.2013.1425
17 Guy GP, Yabroff KR, Ekwueme DU, Virgo KS, Han X, Banegas MP, Soni A, Zheng Z, Chawla N and Geiger AM: Healthcare expenditure burden among non-elderly cancer survivors, 20082012. Am J Prev Med 49(6): S489-497, 2015. PMID: 26590644. DOI: $10.1016 /$ j.amepre.2015.09.002

18 Bernard DS, Farr SL and Fang Z: National estimates of out-ofpocket health care expenditure burdens among nonelderly adults with cancer: 2001 to 2008. J Clin Oncol 29(20): 2821-2826, 2011. PMID: 21632508. DOI: 10.1200/JCO.2010.33.0522

19 Davidoff AJ, Erten M, Shaffer T, Shoemaker JS, Zuckerman IH, Pandya N, Tai MH, Ke X and Stuart B: Out-of-pocket health care expenditure burden for Medicare beneficiaries with cancer. Cancer 119(6): 1257-1265, 2013. PMID: 23225522. DOI: $10.1002 /$ cncr. 27848

20 Langa KM, Fendrick AM, Chernew ME, Kabeto MU, Paisley KL and Hayman JA: Out-of-pocket health-care expenditures among older Americans with cancer. Value Health 7(2): 186-194, 2004. PMID: 15164808. DOI: 10.1111/j.1524-4733.2004.72334.x

21 Nicol GE, Piccirillo JF, Mulsant BH and Lenze EJ: Action at a distance: Geriatric research during a pandemic. J Am Geriatr Soc 68(5): 922-925, 2020. PMID: 32207542. DOI: 10.1111/jgs.16443

Received August 26, 2020

Revised September 9, 2020

Accepted September 10, 2020 